\title{
Existence of solutions for subquadratic convex operator equations at resonance and applications to Hamiltonian systems
}

\author{
Mingliang Song ${ }^{1,2^{*}}$ and Ping Chen ${ }^{2}$
}

\author{
"Correspondence: \\ mlsong2004@163.com \\ 'School of Mathematical Sciences, \\ Nanjing Normal University, Nanjing, \\ Jiangsu 210097, P.R. China \\ ${ }^{2}$ Mathematics and Information \\ Technology School, Jiangsu Second \\ Normal University, Nanjing, Jiangsu \\ 210013, P.R. China
}

\begin{abstract}
This paper investigates the existence of solutions to subquadratic operator equations with convex nonlinearities and resonance by means of the index theory for self-adjoint linear operators developed by Dong and dual least action principle developed by Clarke and Ekeland. Applying the results to subquadratic convex Hamiltonian systems satisfying several boundary value conditions including Bolza boundary value conditions, generalized periodic boundary value conditions and Sturm-Liouville boundary value conditions yield some new theorems concerning the existence of solutions or nontrivial solutions. In particular, some famous results about solutions to subquadratic convex Hamiltonian systems by Mawhin and Willem and Ekeland are special cases of the theorems.
\end{abstract}

MSC: 34B15; 37L50; 47A75; 58E05; 70H05; 70H12

Keywords: Operator equations; Subquadratic; Index theory; Dual least action principle; Convex Hamiltonian systems

\section{Introduction and main results}

Periodic solutions of Hamiltonian systems are important in applications. In recent years, the existence of periodic solutions for Hamiltonian systems has been studied extensively by means of critical point theory and the least action principle, and many interesting results have been obtained. Different solvability hypotheses on the potential are given, such as: the convexity conditions (see $[2,5,7-9,15,16,20]$ ); the subquadratic conditions (see $[5,7,10-13,15-18,20]$ ) and the sublinear conditions (see [19]). In particular, Mawhin and Willem [15, 16] and Ekeland [7] investigated the existence of periodic solutions to convex Hamiltonian systems under subquadratic growth assumptions on the Hamiltonian. In this paper, we will generalize their results to abstract operator equations. Let $X$ be a real infinite-dimensional separable Hilbert space with norm $\|\cdot\|$ and inner product $(\cdot, \cdot)$. Let $A: D(A) \subset X \rightarrow X$ be an unbounded self-adjoint operator with $\sigma(A)=\sigma_{d}(A)=\{\lambda \in \mathbf{R} \mid \lambda$ belongs to the point spectrum of $A\}$. We consider the existence of solutions of the following equation:

$$
A x-B_{1} x \in \partial \Phi(x)
$$

(c) The Author(s) 2020. This article is licensed under a Creative Commons Attribution 4.0 International License, which permits use, sharing, adaptation, distribution and reproduction in any medium or format, as long as you give appropriate credit to the original author(s) and the source, provide a link to the Creative Commons licence, and indicate if changes were made. The images or other third party material in this article are included in the article's Creative Commons licence, unless indicated otherwise in a credit line to the material. If material is not included in the article's Creative Commons licence and your intended use is not permitted by statutory regulation or exceeds the permitted use, you will need to obtain permission directly from the copyright holder. To view a copy of this licence, visit http://creativecommons.org/licenses/by/4.0/. 
where $B_{1} \in \mathcal{L}_{s}(X)$ and $\mathcal{L}_{s}(X)$ denotes the usual space consisting of bounded symmetric operators in $X, v_{A}\left(B_{1}\right) \neq 0$ (see the Appendix for the notation $v_{A}\left(B_{1}\right)$ ), $\Phi: X \rightarrow \mathbf{R}$ is continuous and convex, and $\partial \Phi$ denotes the subdifferential of $\Phi$. Set $\Phi^{*}(y)=\sup _{x \in X}\{(y, x)-\Phi(x)\}$ and $\Phi_{\epsilon}(x)=\Phi(x)+\frac{\epsilon}{2}\|x\|^{2}$ for $\epsilon>0$. Now we use the index $\left(i_{A}(B), \nu_{A}(B)\right) \in \mathbf{Z} \times \mathbf{N}$ defined in $[5,6]$ (see the Appendix) for all $B \in \mathcal{L}_{s}(X)$ to formulate our main results.

\section{Theorem 1.1 Assume that $\Phi \in C(X, \mathbf{R})$ and satisfies}

$\left(\Phi_{1}\right) \Phi$ is convex;

$\left(\Phi_{2}\right)$ there exists $l_{0} \in X$ such that for all $x \in X$ one has

$$
\Phi(x) \geq\left(x, l_{0}\right)
$$

$\left(\Phi_{3}\right)$ there exist $B_{2} \in \mathcal{L}_{s}(X), \epsilon_{0}>0$ and $c>0$, such that $B_{2} \geq B_{1}$ and $B_{2}>B_{1}$ with respect to $\operatorname{ker}\left(A-B_{1}\right)$ (see the Appendix for the notation $B_{2} \geq B_{1}$ and $\left.B_{2}>B_{1}\right), v_{A}\left(B_{2}+\epsilon_{0}\right)=0$, $i_{A}\left(B_{2}+\epsilon_{0}\right)=i_{A}\left(B_{1}\right)+v_{A}\left(B_{1}\right)$, and for all $x \in X$ one has

$$
\Phi(x) \leq \frac{1}{2}\left(\left(B_{2}-B_{1}\right) x, x\right)+c
$$

$\left(\Phi_{4}\right) \Phi(x) \rightarrow+\infty$ as $\|x\| \rightarrow \infty$ and $x \in \operatorname{ker}\left(A-B_{1}\right)$.

Then (1.1) has at least one solution $x$ such that $y=\left(A-B_{1}\right) x$ minimizes the dual action

$$
\psi_{1}: \quad R\left(A-B_{1}\right) \rightarrow \mathbf{R}, \quad y \rightarrow-\frac{1}{2}\left(\Lambda^{-1} y, y\right)+\Phi^{*}(y)
$$

provided $\Phi_{\epsilon}^{*} \in C^{1}(X, \mathbf{R})$, where $\Lambda x=\left(A-B_{1}\right) x$ for all $x \in D(A) \cap R\left(A-B_{1}\right)$.

Theorem 1.2 Under the hypotheses in Theorem 1.1, assume, in addition, that

$\left(\Phi_{5}\right) \nabla \Phi(\theta)=\theta, \Phi(\theta)=0$ and there exist $B_{01} \in \mathcal{L}_{s}(X)$ and $\epsilon_{01}>0$ such that $B_{01}-\epsilon_{01} \geq B_{1}$, $i_{A}\left(B_{01}\right)>i_{A}\left(B_{1}\right)+v_{A}\left(B_{1}\right)$, and

$$
\Phi(x) \geq \frac{1}{2}\left(\left(B_{01}-B_{1}\right) x, x\right), \quad \forall x \in X \text { with small }\|x\| .
$$

Then (1.1) has at least one nontrivial solution.

Remark 1 In [5], Theorem 3.1.7, one only assumed that $\left(\Phi_{1}\right)$ and $\left(\Phi_{3}\right)$ hold. The following BVP can serve as a counterexample:

$$
\left\{\begin{array}{l}
\ddot{x}(t)+\pi^{2} x(t)=1, \quad t \in(0,1), \\
x(0)=0=x(1) .
\end{array}\right.
$$

In fact, it is easy to check that Eq. (1.5) satisfies the conditions of Theorem 3.1.7 in [5], but it has no solution, so Theorem 3.1.7 is also incorrect. These show that our results improve Theorem 3.1.7 in [5].

The paper is organized as follows. The proofs of Theorems 1.1-1.2 are given in Sect. 2 and in Sect. 3 we investigate their applications to convex Hamiltonian systems satisfying 
several boundary value conditions including Bolza boundary value conditions, generalized periodic boundary value conditions and Sturm-Liouville boundary value conditions, and we obtain some new theorems on the existence of solutions or nontrivial solutions. In addition, we give some remarks to illustrate that some famous results about solutions to subquadratic convex Hamiltonian systems by Mawhin and Willem [16] and Ekeland [7] are special cases of these results. In the Appendix we recall some useful results concerning the index theory for unbounded linear self-adjoint operator equations from $[5,6]$ used in other sections.

\section{Proofs of the theorems}

In order to prove Theorems 1.1-1.2 we need some lemmas from $[8,16]$.

Lemma 2.1 ([16], Theorem 1.1) If $\varphi: X \rightarrow \mathbf{R}$ is weakly lower semi-continuous on a Hilbert space $X$ and has a bounded minimizing sequence, then $\varphi$ has a minimum on $X$.

Lemma 2.2 ([16], Theorem 1.2) If $X$ is a Hilbert space and $\varphi: X \rightarrow \mathbf{R}$ is lower semicontinuous and convex, then $\varphi$ is weakly lower semi-continuous.

Let $X$ be a Hilbert space, we shall denote by $\Gamma(X)$ the set of all convex lower semicontinuous functions $F: X \rightarrow \mathbf{R}$. The conjugate function $F^{*}$ of $F$ is the function $F^{*}: X \rightarrow \mathbf{R}$ defined by

$$
F^{*}(v)=\sup _{u \in X}\{(v, u)-F(u)\}
$$

We define the subdifferential of a function $F \in \Gamma(X)$ at a point $u \in X$ to be the set

$$
\partial F(u)=\{v \in X: F(w) \geq F(u)+(v, w-u) \text { for all } w \in X\},
$$

and say that $F$ is subdifferentiable at $u$ if $\partial F(u) \neq \emptyset$.

Lemma 2.3 ([8], Proposition 5.1 and Corollary 5.2) If $F \in \Gamma(X)$, the following statements are equivalent:

(i) $v \in \partial F(u)$;

(ii) $F(u)+F^{*}(v)=(v, u)$;

(iii) $u \in \partial F^{*}(v)$.

Lemma 2.4 ([8], Proposition 5.3) If $F: X \rightarrow \mathbf{R}$ is convex and differentiable at $u$, then $\partial F(u)=\{\nabla F(u)\}$.

Proposition 2.5 For any $B_{1} \in \mathcal{L}_{s}(X)$, if $x \in R\left(A-B_{1}\right) \cap D(A) \subset X$, then there exists $\tilde{\lambda}_{0}>0$ such that

$$
\|x\|^{2} \leq \frac{1}{\tilde{\lambda}_{0}^{2}}\left\|\left(A-B_{1}\right) x\right\|^{2}
$$

Proof By Kato-Rellich's theorem ([1], Theorem 6.5.10), $A-B_{1}$ is unbounded self-adjoint. $\sigma(A)=\sigma_{d}(A)$ shows that there is a basis $\left\{e_{i}\right\}_{i=-\infty}^{\infty}$ of $X$ and $\cdots \leq \lambda_{-2} \leq \lambda_{-1} \leq \lambda_{0} \leq \lambda_{1} \leq \lambda_{2} \leq$ 
.. such that $A e_{j}=\lambda_{j} e_{j}, D(A)=\left\{\sum_{j=-\infty}^{\infty} c_{j} e_{j} \mid \sum_{j=-\infty}^{\infty} \lambda_{j}^{2} c_{j}^{2}<\infty\right\}$. Let $\|x\|_{A}^{2}=\|x\|^{2}+\|A x\|^{2}=$ $\sum_{j=-\infty}^{\infty}\left(\lambda_{j}^{2}+1\right) c_{j}^{2}$ for all $x \in D(A)$. If $\left\{x_{j}\right\} \subset D(A)$ such that $\left\{\left\|x_{j}\right\|_{A}\right\}$ is bounded, then $\left\{x_{j}\right\}$ has a convergent subsequence in $X$ and so does $\left\{B_{1} x_{j}\right\}$. This implies that $B_{1}:\left(D(A),\|\cdot\|_{A}\right) \rightarrow X$ is compact and $B_{1}$ is $A$-compact. By Weyl's theorem ([1], Theorem 6.5.21), we can see that $A-B_{1}$ is also an unbounded linear self-adjoint operator in $X$ and $\sigma_{\text {ess }}\left(A-B_{1}\right)=\sigma_{\text {ess }}(A)=\emptyset$, i.e., $\sigma\left(A-B_{1}\right)=\sigma_{d}\left(A-B_{1}\right)$ is unbounded. From Lemma 3.2.1 in [5], we know that $A-$ $B_{1}: D(A) \rightarrow X$ is continuous, $\operatorname{ker}\left(A-B_{1}\right)$ is finite-dimensional, $R\left(A-B_{1}\right)$ is closed and $X=\operatorname{ker}\left(A-B_{1}\right) \oplus R\left(A-B_{1}\right)$. Note that $v_{A}\left(B_{1}\right) \neq 0$, we can assume that there is a basis $\left\{\tilde{e}_{j}\right\}_{j=-\infty}^{\infty}$ of $X$ and $\cdots \leq \tilde{\lambda}_{-2} \leq \tilde{\lambda}_{-1} \leq 0 \leq \tilde{\lambda}_{1} \leq \tilde{\lambda}_{2} \leq \cdots$ such that $\left(A-B_{1}\right) \tilde{e}_{j}=\tilde{\lambda}_{j} \tilde{e}_{j}$. Thus, for all $x=\sum_{\tilde{\lambda}_{j} \neq 0} c_{j} \tilde{e}_{j} \in R\left(A-B_{1}\right) \cap D(A)$, one has

$$
\|x\|^{2}=\sum_{\tilde{\lambda}_{j} \neq 0} c_{j}^{2} \leq \frac{1}{\tilde{\lambda}_{0}^{2}} \sum_{\tilde{\lambda}_{j} \neq 0} \tilde{\lambda}_{j}^{2} c_{j}^{2}=\frac{1}{\tilde{\lambda}_{0}^{2}}\left\|\left(A-B_{1}\right) x\right\|^{2},
$$

where $\tilde{\lambda}_{0}=\min \left\{\left|\tilde{\lambda}_{j}\right| \neq 0\right\}$.

Consider the dual functional $\psi: R\left(A-B_{1}\right) \rightarrow \mathbf{R}$ defined by

$$
\psi(x)=-\frac{1}{2}\left(\Lambda^{-1} x, x\right)+F^{*}(x)
$$

where $F^{*}(x)=\sup _{y \in X}\{(x, y)-F(y)\}$ for every $F \in \Gamma(X), \Lambda=\left.\left(A-B_{1}\right)\right|_{R\left(A-B_{1}\right)}: R\left(A-B_{1}\right) \cap$ $D(A) \rightarrow R\left(A-B_{1}\right)$ and for the existence of $\Lambda^{-1}$, we refer to the Appendix.

Proposition 2.6 Let $F: X \rightarrow \mathbf{R}$ be convex and continuous and assume $F^{*} \in C^{1}(X, \mathbf{R})$. Then the dual functional $\psi: R\left(A-B_{1}\right) \rightarrow \mathbf{R}$ defined by (2.1) is continuously differentiable and, if $y \in R\left(A-B_{1}\right)$ is a critical point of $\psi$, the element $x$ defined by $x=\Lambda^{-1} y+\xi$ for some $\xi \in \operatorname{ker}\left(A-B_{1}\right)$ satisfies

$$
\left(A-B_{1}\right) x \in \partial F(x) .
$$

Proof If $y \in R\left(A-B_{1}\right)$ is a critical point of $\psi$, Lemma 2.4 implies that, for all $h \in R\left(A-B_{1}\right)$, one has

$$
0=-\left(\Lambda^{-1} y, h\right)+\left(\nabla F^{*}(y), h\right)
$$

It is then easy to see that $-\Lambda^{-1} y+\nabla F^{*}(y) \in \operatorname{ker}\left(A-B_{1}\right)$ via $X=\operatorname{ker}\left(A-B_{1}\right) \oplus R\left(A-B_{1}\right)$, and there is $\xi \in \operatorname{ker}\left(A-B_{1}\right)$ such that

$$
-\Lambda^{-1} y+\nabla F^{*}(y)=\xi
$$

Setting $x=\Lambda^{-1} y+\xi=\nabla F^{*}(y)$, we have $x \in X,\left(A-B_{1}\right) x=\Lambda x=y$ and, by duality, $y \in \partial F(x)$. Thus

$$
\left(A-B_{1}\right) x \in \partial F(x) .
$$

The proof is complete. 
Proof of Theorem 1.1 We divide the proof into three steps.

Step 1. Existence of a solution for a perturbed problem. Let

$$
\Phi_{\epsilon}: \quad X \rightarrow \mathbf{R}, \quad x \rightarrow \frac{\epsilon}{2}\|x\|^{2}+\Phi(x),
$$

where $\epsilon \in\left(0, \epsilon_{0}\right)$. From $\left(\Phi_{2}\right)$ and $\left(\Phi_{3}\right)$, we can obtain

$$
\frac{\epsilon}{2}\|x\|^{2}-\left\|l_{0}\right\|\|x\| \leq \Phi_{\epsilon}(x) \leq \frac{\epsilon_{0}}{2}\|x\|^{2}+\frac{1}{2}\left(\left(B_{2}-B_{1}\right) x, x\right)+c,
$$

hence

$$
\frac{\epsilon\|x\|^{2}}{4}-\frac{\left\|l_{0}\right\|^{2}}{\epsilon} \leq \Phi_{\epsilon}(x) \leq \frac{\epsilon_{0}+\left\|B_{2}-B_{1}\right\|}{2}\|x\|^{2}+c .
$$

So that by Proposition 2.6, the perturbed dual functional

$$
\psi_{\epsilon}(y)=-\frac{1}{2}\left(\Lambda^{-1} y, y\right)+\Phi_{\epsilon}^{*}(y)
$$

is continuously differentiable on $R\left(A-B_{1}\right)$ and if $y_{\epsilon} \in R\left(A-B_{1}\right)$ is a critical point of $\psi_{\epsilon}$, then the element $x_{\epsilon}$ defined by

$$
x_{\epsilon}=\Lambda^{-1} y_{\epsilon}+\xi
$$

for some $\xi \in \operatorname{ker}\left(A-B_{1}\right)$ is a solution of $\left(A-B_{1}\right) x=\nabla \Phi_{\epsilon}(x)$, i.e.,

$$
\left(A-B_{1}\right) x_{\epsilon}-\epsilon x_{\epsilon} \in \partial \Phi\left(x_{\epsilon}\right)
$$

and the relation

$$
\left(A-B_{1}\right) x_{\epsilon}=y_{\epsilon}
$$

holds. Again by (2.4) and (2.1), we can obtain

$$
\Phi_{\epsilon}^{*}(y) \geq \frac{1}{2}\left(y,\left(B_{2}+\epsilon_{0}-B_{1}\right)^{-1} y\right)-c
$$

and

$$
\begin{aligned}
\psi_{\epsilon}(y) & \geq-\frac{1}{2}\left(y, \Lambda^{-1} y\right)+\frac{1}{2}\left(y,\left(B_{2}+\epsilon_{0}-B_{1}\right)^{-1} y\right)-c \\
& =\frac{1}{2} q_{A,\left(B_{2}+\epsilon_{0}\right) \mid B_{1}}(y, y)-c, \quad \forall y \in R\left(A-B_{1}\right) .
\end{aligned}
$$

From Proposition A.4, Lemma A.6 and $\left(\Phi_{3}\right)$, we have $v_{A}\left(B_{1}\right)=i_{A}\left(B_{2}+\epsilon_{0}\right)-i_{A}\left(B_{1}\right)=$ $I_{A}\left(B_{1}, B_{2}+\epsilon_{0}\right)=i_{A}\left(\left(B_{2}+\epsilon_{0}\right) \mid B_{1}\right)+v_{A}\left(B_{1}\right)$, which implies that $i_{A}\left(\left(B_{2}+\epsilon_{0}\right) \mid B_{1}\right)=0$ and $E_{A}^{-}\left(\left(B_{2}+\epsilon_{0}\right) \mid B_{1}\right)=\{\theta\}$. By (1) of Lemma A.6, we have $v_{A}\left(\left(B_{2}+\epsilon_{0}\right) \mid B_{1}\right)=v_{A}\left(B_{2}+\epsilon_{0}\right)=0$ and $E_{A}^{0}\left(\left(B_{2}+\epsilon_{0}\right) \mid B_{1}\right)=\{\theta\}$. By (A.2) of the Appendix, we have

$$
R\left(A-B_{1}\right)=E_{A}^{-}\left(\left(B_{2}+\epsilon_{0}\right) \mid B_{1}\right) \oplus E_{A}^{0}\left(\left(B_{2}+\epsilon_{0}\right) \mid B_{1}\right) \oplus E_{A}^{+}\left(\left(B_{2}+\epsilon_{0}\right) \mid B_{1}\right) .
$$


Thus we can see that $R\left(A-B_{1}\right)=E_{A}^{+}\left(\left(B_{2}+\epsilon_{0}\right) \mid B_{1}\right)$, which implies that $\psi_{\epsilon}(u) \rightarrow+\infty$ as $\|u\| \rightarrow+\infty$ and $\psi_{\epsilon}(u)$ is bounded from below. Assume that $\psi_{\epsilon}\left(y_{j}\right) \rightarrow \inf \psi_{\epsilon}$; then $\psi_{\epsilon}\left(y_{j}\right)$ is bounded and $\left\|y_{j}\right\|$ is also bounded. Now $\psi_{\epsilon, 1}(y)=\Phi_{\epsilon}^{*}(y)$ is weakly lower semi-continuous on $R\left(A-B_{1}\right)$ by Lemma 2.2 and $\psi_{\epsilon, 2}(y)=-\frac{1}{2}\left(y, \Lambda^{-1} y\right)$ is weakly continuous on $R\left(A-B_{1}\right)$ by the compactness of $\Lambda^{-1}$. Thus $\psi_{\epsilon}=\psi_{\epsilon, 1}+\psi_{\epsilon, 2}$ is weakly lower semi-continuous, and by Lemma 2.1, has a minimum at some point $y_{\epsilon} \in R\left(A-B_{1}\right)$.

Step 2. A posteriori estimates on $x_{\epsilon}$. From the obvious inequality $\Phi(x) \leq \Phi_{\epsilon}(x)$ we deduce $\Phi_{\epsilon}^{*}(y) \leq \Phi^{*}(y)$. Thus, we can obtain

$$
\frac{1}{2} q_{A,\left(B_{2}+\epsilon_{0}\right) \mid B_{1}}\left(y_{\epsilon}, y_{\epsilon}\right)-c \leq \psi_{\epsilon}\left(y_{\epsilon}\right) \leq \psi_{\epsilon}(y) \leq-\frac{1}{2}\left(\Lambda^{-1} y, y\right)+\Phi^{*}(y)=\psi_{1}(y),
$$

for all $y \in R\left(A-B_{1}\right)$, which implies that $\psi_{\epsilon}\left(y_{\epsilon}\right) \leq \psi_{1}\left(y_{0}\right)=c_{1}<+\infty$ and $\left\|y_{\epsilon}\right\| \leq c_{2}$ with $c_{2}$ independent of $\epsilon$. By $y_{\epsilon}=\Lambda x_{\epsilon}$, we know that

$$
\left\|\Lambda \tilde{x}_{\epsilon}\right\|=\left\|\Lambda x_{\epsilon}\right\| \leq c_{2}
$$

where $\tilde{x}_{\epsilon}=x_{\epsilon}-\bar{x}_{\epsilon}, \bar{x}_{\epsilon} \in \operatorname{ker}\left(A-B_{1}\right)$. Proposition 2.5 implies that $\left\|\tilde{x}_{\epsilon}\right\| \leq c_{3}$. From the convexity of $\Phi$, hypothesis $\left(\Phi_{3}\right)$ and (2.5) we have

$$
\begin{aligned}
\Phi\left(\frac{\bar{x}_{\epsilon}}{2}\right) & =\Phi\left(\frac{x_{\epsilon}}{2}-\frac{\tilde{x}_{\epsilon}}{2}\right) \leq \frac{1}{2} \Phi\left(x_{\epsilon}\right)+\frac{1}{2} \Phi\left(-\tilde{x}_{\epsilon}\right) \\
& \leq \frac{1}{2}\left(\partial \Phi\left(x_{\epsilon}\right), x_{\epsilon}\right)+\frac{1}{2} \Phi(\theta)+\frac{1}{4}\left\|B_{2}-B_{1}\right\| \cdot\left\|\tilde{x}_{\epsilon}\right\|^{2}+\frac{c}{2} \\
& \leq \frac{1}{2}\left(\left(A-B_{1}\right) x_{\epsilon}, x_{\epsilon}\right)-\frac{\epsilon}{2}\left\|x_{\epsilon}\right\|^{2}+\frac{1}{4}\left\|B_{2}-B_{1}\right\| \cdot\left\|\tilde{x}_{\epsilon}\right\|^{2}+c \\
& \leq \frac{1}{2}\left\|\Lambda^{-1}\right\| \cdot\left\|\Lambda x_{\epsilon}\right\|^{2}+\frac{1}{4}\left\|B_{2}-B_{1}\right\| \cdot\left\|\tilde{x}_{\epsilon}\right\|^{2}+c \\
& \leq c_{4}
\end{aligned}
$$

due to the boundedness of $B_{1}, B_{2}$ and $\Lambda^{-1}$. By assumption $\left(\Phi_{4}\right),\left\|\bar{x}_{\epsilon}\right\| \leq c_{5}$. Then

$$
\left\|x_{\epsilon}\right\| \leq\left\|\tilde{x}_{\epsilon}\right\|+\left\|\bar{x}_{\epsilon}\right\| \leq c_{3}+c_{5}=c_{6} .
$$

Step 3. Existence of a solution for the original problem. Since $\left\|\Lambda \tilde{x}_{\epsilon}\right\| \leq c_{2}$ and $\left\|\bar{x}_{\epsilon}\right\| \leq c_{5}$, there is a sequence $\left\{\varepsilon_{n}\right\}$ in $\left(0, \epsilon_{0}\right)$ tending to 0 and some $\tilde{x} \in R\left(A-B_{1}\right), \bar{x} \in \operatorname{ker}\left(A-B_{1}\right)$ such that $\Lambda \tilde{x}_{\epsilon_{n}} \rightarrow \Lambda \tilde{x}$ and $\bar{x}_{\epsilon_{n}} \rightarrow \bar{x}$ in $X$. Moreover, by the compactness of $\Lambda^{-1}$, we have

$$
\tilde{x}_{\epsilon_{n}} \rightarrow \tilde{x}, \quad x_{\epsilon_{n}}=\tilde{x}_{\epsilon_{n}}+\bar{x}_{\epsilon_{n}} \rightarrow x=\tilde{x}+\bar{x} \in X .
$$

Again note that $\Lambda x_{\epsilon}=y_{\epsilon}$ and $\left\|\Lambda x_{\epsilon}\right\| \leq c_{2}$, we have $\Lambda^{-1} y_{\epsilon}=x_{\epsilon}-\bar{x}_{\epsilon}$ and $y_{\epsilon_{n}} \rightarrow y$ in $R\left(A-B_{1}\right)$, so that $\Lambda^{-1} y_{\epsilon_{n}}$ converges to

$$
\Lambda^{-1} y=x-\bar{x}
$$

From Proposition 2.6, we can see that

$$
\Lambda x_{\epsilon_{n}}-\epsilon_{n} x_{\epsilon_{n}} \in \partial \Phi\left(x_{\epsilon_{n}}\right) .
$$


By definition,

$$
\Phi(u) \geq \Phi\left(x_{\epsilon_{n}}\right)+\left(\Lambda x_{\epsilon_{n}}-\epsilon_{n} x_{\epsilon_{n}}, u-x_{\epsilon_{n}}\right), \quad \forall u \in X .
$$

Taking the limit yields

$\Lambda x \in \partial \Phi(x)$

which shows that $x \in X$ is a solution of (1.1).

Finally, as $\Phi_{\epsilon}^{*}(y) \leq \Phi^{*}(y)$, we have, for all $h \in R\left(A-B_{1}\right)$

$$
\psi_{\epsilon_{n}}\left(y_{\epsilon_{n}}\right) \leq \psi_{\epsilon_{n}}(h) \leq \psi(h)
$$

Now, by the duality between $x_{\epsilon_{n}}$ and $y_{\epsilon_{n}}$, we have

$$
\begin{aligned}
\psi_{\epsilon_{n}}\left(y_{\epsilon_{n}}\right) & =-\frac{1}{2}\left(\Lambda^{-1} y_{\epsilon_{n}}, y_{\epsilon_{n}}\right)+\Phi_{\epsilon_{n}}^{*}\left(y_{\epsilon_{n}}\right) \\
& =-\frac{1}{2}\left(\Lambda^{-1} y_{\epsilon_{n}}, y_{\epsilon_{n}}\right)+\left(x_{\epsilon_{n}}, y_{\epsilon_{n}}\right)-\Phi_{\epsilon_{n}}\left(x_{\epsilon_{n}}\right) \\
& =-\frac{1}{2}\left(\Lambda^{-1} y_{\epsilon_{n}}, y_{\epsilon_{n}}\right)+\left(x_{\epsilon_{n}}, y_{\epsilon_{n}}\right)-\Phi\left(x_{\epsilon_{n}}\right)-\frac{\epsilon_{n}}{2}\left\|x_{\epsilon_{n}}\right\|^{2} .
\end{aligned}
$$

It follows from (1.1) and (2.7) that

$$
y=\left(A-B_{1}\right) x \in \partial \Phi(x) .
$$

Letting $n \rightarrow \infty$ in (2.9), by (2.10) and Lemma 2.3, we obtain

$$
\begin{aligned}
\lim _{n \rightarrow \infty} \psi_{\epsilon_{n}}\left(y_{\epsilon_{n}}\right) & =-\frac{1}{2}\left(\Lambda^{-1} y, y\right)+(x, y)-\Phi(x) \\
& =-\frac{1}{2}\left(\Lambda^{-1} y, y\right)+\Phi^{*}(y)=\psi(y) .
\end{aligned}
$$

Thus $\psi(y) \leq \psi(h)$ for all $h \in R\left(A-B_{1}\right)$ and the proof is complete.

Proof of Theorem 1.2 From Theorem 1.1 we know that there is $y_{0} \in R\left(A-B_{1}\right)$ such that $x=\Lambda^{-1} y_{0}+\bar{x}$ for some $\bar{x} \in \operatorname{ker}\left(A-B_{1}\right)$ is a solution of $(1.1)$, and

$$
\psi\left(y_{0}\right) \leq \psi(h), \quad \forall h \in R\left(A-B_{1}\right)
$$

If $\theta \in \partial \Phi(\theta)$, then $\theta$ is a solution of (1.1). We can prove that $y_{0} \neq \theta$ under assumption $\left(\Phi_{5}\right)$. In fact, by (1.4), we can obtain

$$
\begin{aligned}
\psi(h) & \leq-\frac{1}{2}\left(h, \Lambda^{-1} h\right)+\frac{1}{2}\left(h,\left(B_{01}-B_{1}\right)^{-1} h\right) \\
& =\frac{1}{2} q_{A, B_{01} \mid B_{1}}(h, h) \quad \text { as } h \rightarrow \theta .
\end{aligned}
$$

From Proposition A.4, Lemma A.6 and $\left(\Phi_{5}\right)$, we have $I_{A}\left(B_{1}, B_{01}\right)=i_{A}\left(B_{01} \mid B_{1}\right)+v_{A}\left(B_{1}\right)=$ $i_{A}\left(B_{01}\right)-i_{A}\left(B_{1}\right)>v_{A}\left(B_{1}\right)$, which shows that $i_{A}\left(B_{01} \mid B_{1}\right)>0$ and $\operatorname{dim}\left(E_{A}^{-}\left(B_{01} \mid B_{1}\right)\right) \geq 1$ by (A.2) 
in the Appendix. So, for any $h \in E_{A}^{-}\left(B_{01} \mid B_{1}\right) \backslash\{\theta\}$ small enough, we have $\psi(h)<0=\psi(\theta)$. Hence $y_{0} \neq \theta$ and $x=\Lambda^{-1} y_{0}+\bar{x} \neq \theta$. This completes the proof.

\section{Applications to convex Hamiltonian systems}

In this section, we consider the applications of the main results to convex Hamiltonian systems. For systematic research of Hamiltonian systems, we refer to the excellent books $[7,14]$.

\subsection{First order Hamiltonian systems satisfying Bolza boundary value conditions}

As a first example, we now consider a BVP for a the nonlinear Hamiltonian systems (3.1)(3.3):

$$
\begin{aligned}
& -J \dot{x}-\bar{B}_{1}(t) x-H^{\prime}(t, x)=0, \quad t \in[0,1] \\
& x_{1}(0) \cos \alpha+x_{2}(0) \sin \alpha=0, \\
& x_{1}(1) \cos \beta+x_{2}(1) \sin \beta=0,
\end{aligned}
$$

where $J=\left(\begin{array}{cc}0 & -I_{n} \\ I_{n} & 0\end{array}\right), \bar{B}_{1} \in L^{\infty}\left([0,1], \mathcal{L}_{s}\left(\mathbf{R}^{2 n}\right)\right)=\left\{B(t)=\left(b_{j k}\right)_{2 n \times 2 n} \mid b_{j k}(t)=b_{k j}(t), t \in[0,1]\right.$, $\left.b_{j k}(t) \in L^{\infty}([0,1])\right\}$ with $v_{\alpha, \beta}^{f}\left(\bar{B}_{1}\right) \neq 0$ and $I_{n}$ is the identity matrix on $\mathbf{R}^{n}, 0 \leq \alpha<\pi$, $0<\beta \leq \pi, x=\left(x_{1}, x_{2}\right) \in \mathbf{R}^{n} \times \mathbf{R}^{n}, k \in \mathbf{Z}, H:[0,1] \times \mathbf{R}^{2 n} \rightarrow \mathbf{R}^{2 n}$ is differentiable and $H^{\prime}(t, x)$ is the gradient of $H$ with respect to $x$.

From Theorems 1.1-1.2, we have the following theorem.

Theorem 3.1 Assume that $H \in C^{1}\left(\mathbf{R}^{2 n}, \mathbf{R}\right)$ and satisfies

$\left(H_{1}\right) H(t, x)$ is convex in $x \in \mathbf{R}^{2 n}$ for almost every $t \in[0,1]$;

$\left(H_{2}\right)$ there exists $l_{0} \in L^{2}\left([0,1], \mathbf{R}^{2 n}\right)$ such that for all $x \in \mathbf{R}^{2 n}$ one has

$$
H(t, x) \geq\left(x, l_{0}(t)\right)
$$

$\left(H_{3}\right)$ there exist $\bar{B}_{2} \in L^{\infty}\left([0,1], \mathcal{L}_{s}\left(\mathbf{R}^{2 n}\right)\right)$ and $\gamma \in L^{1}\left([0,1], \mathbf{R}^{+}\right)$, such that $\bar{B}_{2} \geq \bar{B}_{1}$, $v_{\alpha, \beta}^{f}\left(\bar{B}_{2}\right)=0, i_{\alpha, \beta}^{f}\left(\bar{B}_{2}\right)=i_{\alpha, \beta}^{f}\left(\bar{B}_{1}\right)+v_{\alpha, \beta}^{f}\left(\bar{B}_{1}\right)$, and for every $x \in \mathbf{R}^{2 n}$ and a.e. $t \in[0,1]$ one has

$$
H(t, x) \leq \frac{1}{2}\left(\left(\bar{B}_{2}(t)-\bar{B}_{1}(t)\right) x, x\right)+\gamma(t)
$$

$\left(H_{4}\right) \int_{0}^{1} H(t, x) d t \rightarrow+\infty$ as $\|x\|_{L^{2}} \rightarrow \infty$ and $x \in \operatorname{ker}\left(A_{1}-\bar{B}_{1}\right)$.

Then (3.1)-(3.3) has at least one solution.

Proof Let $X=L^{2}\left([0,1], \mathbf{R}^{2 n}\right), D\left(A_{1}\right)=\left\{x \in H^{1}\left([0,1], \mathbf{R}^{2 n}\right) \mid x\right.$ satisfies (3.2)-(3.3) $\}$. Define $A_{1}: D\left(A_{1}\right) \rightarrow X$ by $\left(A_{1} x\right)(t)=-J \dot{x}(t)$. It is easy to see that $A_{1}$ is self-adjoint in $X$ and $\sigma\left(A_{1}\right)=\sigma_{d}\left(A_{1}\right)=\{\beta-\alpha+k \pi \mid k \in \mathbf{Z}\}$.

Set $H_{\epsilon}(t, x)=\frac{\epsilon}{2}|x|^{2}+H(t, x), \Phi(x)=\int_{0}^{1} H(t, x(t)) d t$, and $\Phi_{\epsilon}(x)=\int_{0}^{1} H_{\epsilon}(t, x(t)) d t$ for $x \in X$. $\Phi$ and $\Phi_{\epsilon}$ are convex because of the convexity of $H$ with respect to $x$. We have the following proposition. 
Proposition 3.2 ([16], Proposition 2.2) Let $F \in C^{1}\left(\mathbf{R}^{n}, \mathbf{R}\right)$ be a convex function such that, for some $\alpha>0, b_{1} \geq 0, b_{2} \geq 0$, one has

$$
-b_{1} \leq F(u) \leq \frac{\alpha}{2}|u|^{2}+b_{2}
$$

whenever $u \in \mathbf{R}^{n}$. Then

$$
\left|F^{\prime}(u)\right| \leq 2 \alpha\left[|u|+b_{1}+b_{2}\right]
$$

for $u \in \mathbf{R}^{n}$.

By $\left(H_{2}\right)-\left(H_{3}\right)$,

$$
\frac{\epsilon}{4}|x|^{2}-\frac{1}{\epsilon}\left|l_{0}(t)\right|^{2} \leq H_{\epsilon}(t, x) \leq \frac{1}{2}(c+\epsilon)|x|^{2}+|\gamma(t)|
$$

for all $x \in \mathbf{R}^{2 n}$ and a.e. $t \in[0,1]$. So $\Phi_{\epsilon} \in C^{1}(X, \mathbf{R})$ via Proposition 3.2. Moreover,

$$
\frac{1}{2(c+\epsilon)}|y|^{2}-|\gamma(t)| \leq H_{\epsilon}^{*}(t, y) \leq \frac{1}{\epsilon}\left(|y|^{2}+\left|l_{0}(t)\right|^{2}\right)
$$

for all $y \in \mathbf{R}^{2 n}$ and a.e. $t \in[0,1]$. By ([16], Proposition 2.4$), H_{\epsilon}^{*}(t, \cdot) \in C^{1}\left(\mathbf{R}^{2 n}, \mathbf{R}\right)$ for a.e. $t \in[0,1]$. And from ([7], pages 93-96),

$$
\Phi_{\epsilon}^{*}(y)=\int_{0}^{1} H_{\epsilon}^{*}(t, y(t)) d t
$$

for $y \in X$. Hence, $\Phi_{\epsilon}^{*} \in C^{1}(X, \mathbf{R})$ via Proposition 3.2 again.

$\left(\Phi_{1}\right)$ follows from $\left(H_{1}\right)$ and obviously, $\left(\Phi_{2}\right)$ follows from $\left(H_{2}\right),\left(\Phi_{4}\right)$ follows from $\left(H_{4}\right)$. We need only to show that $\left(\Phi_{3}\right)$ follows from $\left(H_{3}\right)$. In fact, for non-zero $x \in \operatorname{ker}\left(A_{1}-\bar{B}_{1}\right), x=$ $x(t)$ is a non-zero solution of $-J \dot{x}-\bar{B}_{1}(t) x=0$ and (3.2)-(3.3). So $x=x(t)$ has at most finitely many zeros on $[0,1]$, and $\left(\bar{B}_{2} x, x\right)>\left(\bar{B}_{1} x, x\right)$. Moreover, from Proposition 3.3, condition $\left(H_{3}\right)$ and Proposition A.8 (see the Appendix), we know that there exists $\epsilon_{0}>0$ such that $v_{\alpha, \beta}^{f}\left(B_{2}+\epsilon_{0} I_{2 n}\right)=0, i_{\alpha, \beta}^{f}\left(B_{2}+\epsilon_{0} I_{2 n}\right)=i_{\alpha, \beta}^{f}\left(B_{1}\right)+v_{\alpha, \beta}^{f}\left(B_{1}\right)$, which implies that $\left(\Phi_{3}\right)$ holds. The proof is complete.

Proposition 3.3 ([3], Proposition 2.13) For any $B \in L^{\infty}\left([0,1], \mathcal{L}_{s}\left(\mathbf{R}^{2 n}\right)\right)$, there exists $\epsilon_{0}>0$ such that for any $\epsilon \in\left(0, \epsilon_{0}\right]$ we have

$$
\begin{aligned}
& v_{\alpha, \beta}^{f}\left(B+\epsilon I_{2 n}\right)=0=v_{\alpha, \beta}^{f}\left(B-\epsilon I_{2 n}\right), \\
& i_{\alpha, \beta}^{f}\left(B-\epsilon I_{2 n}\right)=i_{\alpha, \beta}^{f}(B), \\
& i_{\alpha, \beta}^{f}\left(B+\epsilon I_{2 n}\right)=i_{\alpha, \beta}^{f}(B)+v_{\alpha, \beta}^{f}(B) .
\end{aligned}
$$

In particular, if $v_{\alpha, \beta}^{f}(B)=0$, we have $i_{\alpha, \beta}^{f}\left(B+\epsilon I_{2 n}\right)=i_{\alpha, \beta}^{f}(B)$ for all $\epsilon \in\left(0, \epsilon_{0}\right]$.

Theorem 3.4 Under the conditions in Theorem 3.1, assume, in addition, that 
$\left(H_{5}\right) H^{\prime}(t, \theta)=\theta, H(t, \theta)=0$ and there exists $B_{01} \in L^{\infty}\left([0,1], \mathcal{L}_{s}\left(\mathbf{R}^{2 n}\right)\right)$ such that $B_{01} \geq \bar{B}_{1}$, $i_{\alpha, \beta}^{f}\left(B_{01}\right)>i_{\alpha, \beta}^{f}\left(\bar{B}_{1}\right)+v_{\alpha, \beta}^{f}\left(\bar{B}_{1}\right)$, and

$$
H(t, x) \geq \frac{1}{2}\left(\left(B_{01}-\bar{B}_{1}\right) x, x\right), \quad \forall x \in \mathbf{R}^{2 n} \text { with small }|x| .
$$

Then (3.1)-(3.3) has at least one nontrivial solution.

Proof It is obvious that $\left(H_{5}\right)$ implies $\left(\Phi_{5}\right)$ and it remains to apply Theorems 1.1-1.2.

\subsection{First order Hamiltonian systems satisfying generalized periodic boundary value conditions}

As a second example, consider the nonlinear Hamiltonian systems:

$$
\begin{aligned}
& -J \dot{x}-\bar{B}_{1} x-H^{\prime}(t, x)=0, \quad t \in[0,1] ; \\
& x(1)=P x(0),
\end{aligned}
$$

where $\bar{B}_{1} \in L^{\infty}\left([0,1], \mathcal{L}_{s}\left(\mathbf{R}^{2 n}\right)\right)$ with $v_{P}^{f}\left(B_{1}\right) \neq 0, P$ is symplectic, i.e. $P^{T} J P=J, H:[0,1] \times$ $\mathbf{R}^{2 n} \rightarrow \mathbf{R}^{2 n}$ is differentiable and $H^{\prime}(t, x)$ is the gradient of $H$ with respect to $x$.

Theorem 3.5 Assume that $H \in C^{1}\left(\mathbf{R}^{2 n}, \mathbf{R}\right)$ and satisfies $\left(H_{1}\right),\left(H_{2}\right)$ and

$\left(H_{3,1}\right)$ there exist $\bar{B}_{2} \in L^{\infty}\left([0,1], \mathcal{L}_{s}\left(\mathbf{R}^{2 n}\right)\right)$ and $\gamma \in L^{1}\left([0,1], \mathbf{R}^{+}\right)$, such that $\bar{B}_{2} \geq \bar{B}_{1}$, $v_{P}^{f}\left(\bar{B}_{2}\right)=0, i_{P}^{f}\left(\bar{B}_{2}\right)=i_{P}^{f}\left(\bar{B}_{1}\right)+v_{P}^{f}\left(\bar{B}_{1}\right)$, and for every $x \in \mathbf{R}^{2 n}$ and a.e. $t \in[0,1]$ one has (3.5) holds.

$\left(H_{4,1}\right) \int_{0}^{1} H(t, x) d t \rightarrow+\infty$ as $\|x\|_{L^{2}} \rightarrow \infty$ and $x \in \operatorname{ker}\left(A_{2}-\bar{B}_{1}\right)$.

Then (3.7)-(3.8) has at least one solution.

Proof Let $X=L^{2}\left([0,1], \mathbf{R}^{2 n}\right), D\left(A_{2}\right)=\left\{x \in H^{1}\left([0,1], \mathbf{R}^{2 n}\right) \mid x\right.$ satisfies (3.8) $\}$. Define $A_{2}$ : $D\left(A_{2}\right) \rightarrow X$ by $\left(A_{2} x\right)(t)=-J \dot{x}(t)$. It is easy to check that $A_{2}$ is self-adjoint in $X$ and $\sigma\left(A_{2}\right)=\sigma_{d}\left(A_{2}\right)=\left\{\mu_{i}+2 j \pi \mid i=1,2, \ldots, m ; j \in \mathbf{Z}\right\}$, where $\left\{\mu_{i}\right\}_{i=1}^{m} \subseteq(0,2 \pi]$ are such that, for some non-zero $\xi_{i} \in \mathbf{R}^{2 n},\left(e^{-J \mu_{i}} P-I_{2 n}\right) \xi_{i}=0$ and $\left(\xi_{i}, \xi_{j}\right)_{\mathbf{R}^{2 n}}=0$ if $\mu_{i}=\mu_{j}$ for $i \neq j$.

Set $\Phi(x)=\int_{0}^{1} H(t, x) d t$ for $x \in X$. Because of Proposition 3.2 and Proposition 3.6 and Proposition A.10 the rest of the proof of Theorem 3.5 is similar to the one of Theorem 3.1.

Proposition 3.6 ([4], Proposition 2.13) For any $B \in L^{\infty}\left([0,1], \mathcal{L}_{s}\left(\mathbf{R}^{2 n}\right)\right)$, there exists $\epsilon_{0}>0$ such that for any $\epsilon \in\left(0, \epsilon_{0}\right]$ we have

$$
\begin{aligned}
& v_{P}^{f}\left(B+\epsilon I_{2 n}\right)=0=v_{P}^{f}\left(B-\epsilon I_{2 n}\right), \\
& i_{P}^{f}\left(B-\epsilon I_{2 n}\right)=i_{P}^{f}(B), \\
& i_{P}^{f}\left(B+\epsilon I_{2 n}\right)=i_{P}^{f}(B)+v_{P}^{f}(B) .
\end{aligned}
$$

In particular, if $v_{P}^{f}(B)=0$, we have $i_{P}^{f}\left(B+\epsilon I_{2 n}\right)=i_{P}^{f}(B)$ for all $\epsilon \in\left(0, \epsilon_{0}\right]$.

Remark 2 Note that $L^{4}\left([0,1], \mathbf{R}^{2 n}\right) \subset L^{2}\left([0,1], \mathbf{R}^{2 n}\right), L^{2}\left([0,1], \mathbf{R}^{+}\right) \subset L^{1}\left([0,1], \mathbf{R}^{+}\right)$. If $P=I_{2 n}$, $T=1, \bar{B}_{1}=0 I_{2 n}, \bar{B}_{2}=\alpha_{0} I_{2 n}<2 \pi I_{2 n}, l_{0} \in L^{4}\left([0,1], \mathbf{R}^{2 n}\right)$ and $\gamma \in L^{2}\left([0,1], \mathbf{R}^{+}\right)$, then Theorem 3.5 reduces to ([16], Theorem 3.1; [7], Proposition III.2.2). Moreover, since $P=I_{2 n}$, 
$T=1, \bar{B}_{2}=\bar{B}_{1}+\epsilon I_{2 n}$, where $\epsilon>0$ is small enough, Theorem 3.5 reduces to ([7], Proposition III.2.3). Clearly, our result generalizes Theorem 3.1 in [16] and Propositions III.2.2III.2.3 in [7].

Corollary 3.7 Assume that $H \in C^{1}\left(\mathbf{R}^{2 n}, \mathbf{R}\right)$ and there exists $\bar{B}_{2} \in L^{\infty}\left([0,1], \mathcal{L}_{s}\left(\mathbf{R}^{2 n}\right)\right)$ with $\bar{B}_{2} \geq \bar{B}_{1}$, such that $H(t, x)+\frac{1}{2}\left(\bar{B}_{1}(t) x, x\right)$ is $\left(\bar{B}_{1}, \bar{B}_{2}\right)$-subquadratic at infinity, i.e., $\left(H_{1}\right)$ holds, and there exists $c \in \mathbf{R}$ such that

$$
H(t, x)+\frac{1}{2}\left(\bar{B}_{1}(t) x, x\right) \leq \frac{1}{2}\left(\bar{B}_{2}(t) x, x\right)+c
$$

for all $(t, x)$, and $n\left(\|x\|_{L^{2}}\right) \leq H(t, x)$ with $\frac{n(s)}{s} \rightarrow+\infty$ as $s \rightarrow+\infty$. Let $\gamma_{i}(t)$ with $0 \leq \gamma_{1}(t) \leq$ $\cdots \leq \gamma_{2 n}(t)$ be the eigenvalues of $\bar{B}_{2}(t)-\bar{B}_{1}(t)$, and let $\lambda_{j}$ be the eigenvalues of $A_{2}-\bar{B}_{1}$, and set

$$
\gamma=\max \left\{\gamma_{2 n}(t) \mid t \in[0,1]\right\}, \quad \lambda=\min \left\{\lambda_{j}>0\right\} .
$$

Then (3.7)-(3.8) has at least one solution if $\lambda>\gamma$.

Proof Clearly, since $H(t, x)+\frac{1}{2}\left(\bar{B}_{1}(t) x, x\right)$ is $\left(\bar{B}_{1}, \bar{B}_{2}\right)$-subquadratic at infinity, we can see that the conditions $\left(H_{2}\right),\left(H_{4,1}\right)$, and $(3.5)\left(\mathrm{cf} .\left(H_{3,1}\right)\right)$ hold. It remains to prove that $v_{P}^{f}\left(\bar{B}_{2}\right)=0$ and $i_{P}^{f}\left(\bar{B}_{2}\right)=i_{P}^{f}\left(\bar{B}_{1}\right)+v_{P}^{f}\left(\bar{B}_{1}\right)$. In fact, by the definition of $\gamma$ we have $\bar{B}_{2}(t) \leq \bar{B}_{1}(t)+\gamma I_{2 n}$. Since $\lambda>$ $\gamma, A_{2} x-\bar{B}_{1} x-\mu x=0$ has no nontrivial solutions for any $\mu \in(0, \gamma]$. From Definition A.9 and (2) of Proposition A.4, we know that $i_{P}^{f}\left(\bar{B}_{1}+\gamma I_{2 n}\right)=i_{P}^{f}\left(\bar{B}_{1}\right)+v_{P}^{f}\left(\bar{B}_{1}\right)$. Since $\bar{B}_{1}(t)<\bar{B}_{2}(t) \leq$ $\bar{B}_{1}(t)+\gamma I_{2 n}$, we have $i_{P}^{f}\left(\bar{B}_{1}\right)+v_{P}^{f}\left(\bar{B}_{1}\right) \leq i_{P}^{f}\left(\bar{B}_{2}\right), i_{P}^{f}\left(\bar{B}_{2}\right)+v_{P}^{f}\left(\bar{B}_{2}\right) \leq i_{P}^{f}\left(\bar{B}_{1}+\gamma I_{2 n}\right)$. So $v_{P}^{f}\left(\bar{B}_{2}\right)=0$ and $i_{P}^{f}\left(\bar{B}_{2}\right)=i_{P}^{f}\left(\bar{B}_{1}\right)+v_{P}^{f}\left(\bar{B}_{1}\right)$.

Remark 3 Noticing that Theorem III.2.1 in [7] considers the eigenvalue of operator $-A_{2}+$ $\bar{B}_{1}$, it is easy see that Corollary 3.7 reduces to ([7], Theorem III.2.1) as $P=I_{2 n}, T=1$.

Similarly to Theorem 3.4 , we have the following.

Theorem 3.8 Under the hypotheses of Theorem 3.5, assume, in addition, that

$\left(H_{5,1}\right) H^{\prime}(t, \theta)=\theta, H(t, \theta)=0$ and there exists $B_{01} \in L^{\infty}\left([0,1], \mathcal{L}_{s}\left(\mathbf{R}^{2 n}\right)\right)$ such that $B_{01} \geq$ $\bar{B}_{1}, i_{p}^{f}\left(B_{01}\right)>i_{p}^{f}\left(\bar{B}_{1}\right)+v_{p}^{f}\left(\bar{B}_{1}\right)$, and (3.6) holds.

Then (3.7)-(3.8) has at least one nontrivial solution.

\subsection{Second order Hamiltonian systems satisfying Sturm-Liouville boundary value conditions}

As a third example, consider the BVP (3.9)-(3.11):

$$
\begin{aligned}
& -\left(\Lambda(t) x^{\prime}\right)^{\prime}-\bar{B}_{1} x-V^{\prime}(t, x)=0, \\
& x(0) \cos \alpha-\Lambda(0) x^{\prime}(0) \sin \alpha=0, \\
& x(1) \cos \beta-\Lambda(1) x^{\prime}(1) \sin \beta=0,
\end{aligned}
$$

where $\Lambda \in C\left([0,1], \mathcal{L}_{s}\left(\mathbf{R}^{n}\right)\right), \Lambda(t)$ is positive definite for $t \in[0,1]$, and $\bar{B}_{1} \in L^{\infty}([0,1]$, $\left.\mathcal{L}_{s}\left(\mathbf{R}^{n}\right)\right)$ with $v_{\Lambda, \alpha, \beta}^{s}\left(\bar{B}_{1}\right) \neq 0,0 \leq \alpha<\pi, 0<\beta \leq \pi$. 
Theorem 3.9 Assume that $V \in C^{1}\left(\mathbf{R}^{n}, \mathbf{R}\right)$ and satisfies

$\left(V_{1}\right) H(t, x)$ is convex in $x \in \mathbf{R}^{n}$ for almost every $t \in[0,1]$;

$\left(V_{2}\right)$ there exists $l_{0} \in L^{2}\left([0,1], \mathbf{R}^{n}\right)$ such that for all $x \in \mathbf{R}^{n}$ one has

$$
V(t, x) \geq\left(x, l_{0}(t)\right)
$$

$\left(V_{3}\right)$ there exist $\bar{B}_{2} \in L^{\infty}\left([0,1], \mathcal{L}_{s}\left(\mathbf{R}^{n}\right)\right)$ and $\gamma \in L^{1}\left([0,1], \mathbf{R}^{+}\right)$such that $\bar{B}_{2} \geq \bar{B}_{1}$, $v_{\Lambda, \alpha, \beta}^{s}\left(\bar{B}_{2}\right)=0, i_{\Lambda, \alpha, \beta}^{s}\left(\bar{B}_{2}\right)=i_{\Lambda, \alpha, \beta}^{s}\left(\bar{B}_{1}\right)+v_{\Lambda, \alpha, \beta}^{s}\left(\bar{B}_{1}\right)$, and for every $x \in \mathbf{R}^{n}$ and a.e. $t \in[0,1]$ one has

$$
V(t, x) \leq \frac{1}{2}\left(\left(\bar{B}_{2}-\bar{B}_{1}\right) x, x\right)+\gamma(t)
$$

$\left(V_{4}\right) \int_{0}^{1} V(t, x) d t \rightarrow+\infty$ as $\|x\|_{L^{2}} \rightarrow \infty$ and $x \in \operatorname{ker}\left(A_{3}-\bar{B}_{1}\right)$.

Then (3.9)-(3.11) has at least one solution.

Proof Let $X=L^{2}\left([0,1], \mathbf{R}^{n}\right)$,

$$
D\left(A_{3}\right)=\left\{x \in C^{2}\left([0,1], \mathbf{R}^{n}\right) \mid\left(\Lambda(t) x^{\prime}\right)^{\prime} \in L^{2}\left([0,1], \mathbf{R}^{n}\right), x \text { satisfies (3.10)-(3.11) }\right\} .
$$

Define $A_{3}: D\left(A_{3}\right) \rightarrow X$ by $\left(A_{3} x\right)(t)=-\left(\Lambda(t) x^{\prime}(t)\right)^{\prime}$. Using the results presented in [5, Sect. 3.3], it is possible to show that $A_{3}$ is self-adjoint and $\sigma\left(A_{3}\right)=\sigma_{d}\left(A_{3}\right)$. In particular, if $\Lambda(t)=I_{n}, \alpha=0, \beta=\pi$, we have $\sigma\left(A_{3}\right)=\sigma_{d}\left(A_{3}\right)=\left\{k^{2} \pi^{2} \mid k \in \mathbf{Z}\right\}$.

Set $\Phi(x)=\int_{0}^{1} V(t, x) d t$ for $x \in X$. Because of Proposition 3.2 and Proposition 3.10 and Proposition A.12 the rest of the proof of Theorem 3.9 is similar to Theorem 3.1.

Proposition 3.10 For any $B \in L^{\infty}\left([0,1], \mathcal{L}_{s}\left(\mathbf{R}^{n}\right)\right)$, there exists $\epsilon_{0}>0$ such that for any $\epsilon \in$ $\left(0, \epsilon_{0}\right]$ we have

$$
\begin{aligned}
& v_{\Lambda, \alpha, \beta}^{s}\left(B+\epsilon I_{n}\right)=0=v_{\Lambda, \alpha, \beta}^{s}\left(B-\epsilon I_{n}\right), \\
& i_{\Lambda, \alpha, \beta}^{s}\left(B-\epsilon I_{n}\right)=i_{\Lambda, \alpha, \beta}^{s}(B), \\
& i_{\Lambda, \alpha, \beta}^{s}\left(B+\epsilon I_{n}\right)=i_{\Lambda, \alpha, \beta}^{s}(B)+v_{\Lambda, \alpha, \beta}^{s}(B) .
\end{aligned}
$$

In particular, if $\nu_{\Lambda, \alpha, \beta}^{s}(B)=0$, we have $i_{\Lambda, \alpha, \beta}^{s}\left(B+\epsilon I_{n}\right)=i_{\Lambda, \alpha, \beta}^{s}(B)$ for all $\epsilon \in\left(0, \epsilon_{0}\right]$.

Similarly to Theorem 3.4, we have the following.

Theorem 3.11 Under the hypotheses of Theorem 3.9, assume, in addition, that

$\left(V_{5}\right) V^{\prime}(t, \theta)=\theta, V(t, \theta)=0$ and there exists $B_{01} \in L^{\infty}\left([0,1], \mathcal{L}_{s}\left(\mathbf{R}^{n}\right)\right)$ such that $B_{01} \geq \bar{B}_{1}$, $i_{\Lambda, \alpha, \beta}^{s}\left(B_{01}\right)>i_{\Lambda, \alpha, \beta}^{s}\left(\bar{B}_{1}\right)+v_{\Lambda, \alpha, \beta}^{s}\left(\bar{B}_{1}\right)$, and

$$
V(t, x) \geq \frac{1}{2}\left(\left(B_{01}-\bar{B}_{1}\right) x, x\right) \quad \forall x \in \mathbf{R}^{n} \text { with small }|x| .
$$

Then (3.9)-(3.11) has at least one nontrivial solution. 


\subsection{Second order Hamiltonian systems satisfying generalized periodic boundary value conditions}

Finally, we consider the BVP (3.15)-(3.17):

$$
\begin{aligned}
& -x^{\prime \prime}-\bar{B}_{1} x-V^{\prime}(t, x)=0, \\
& x(1)=M x(0), \\
& x^{\prime}(1)=N x^{\prime}(0),
\end{aligned}
$$

where $\bar{B}_{1} \in L^{\infty}\left([0,1], \mathcal{L}_{s}\left(\mathbf{R}^{n}\right)\right)$ with $v_{M}^{s}\left(\bar{B}_{1}\right) \neq 0, M, N \in G L(n)$ and $M N^{T}=I_{n}$.

Theorem 3.12 Assume that $V \in C^{1}\left(\mathbf{R}^{n}, \mathbf{R}\right)$ and satisfies $\left(V_{1}\right),\left(V_{2}\right)$ and

$\left(V_{3,1}\right)$ there exist $\bar{B}_{2} \in L^{\infty}\left([0,1], \mathcal{L}_{s}\left(\mathbf{R}^{n}\right)\right)$ and $\gamma \in L^{1}\left([0,1], \mathbf{R}^{+}\right)$such that $\bar{B}_{2} \geq \bar{B}_{1}$, $\nu_{M}^{s}\left(\bar{B}_{2}\right)=0, i_{M}^{s}\left(\bar{B}_{2}\right)=i_{M}^{s}\left(\bar{B}_{1}\right)+\nu_{M}^{s}\left(\bar{B}_{1}\right)$, and for every $x \in \mathbf{R}^{n}$ and a.e. $t \in[0,1](3.13)$ holds;

$\left(V_{4,1}\right) \int_{0}^{1} V(t, x) d t \rightarrow+\infty$ as $\|x\|_{L^{2}} \rightarrow \infty$ and $x \in \operatorname{ker}\left(A_{4}-\bar{B}_{1}\right)$.

Then (3.15)-(3.17) has at least one solution.

Proof Let $X=L^{2}\left([0,1], \mathbf{R}^{n}\right)$,

$$
D\left(A_{4}\right)=\left\{x \in C^{2}\left([0,1], \mathbf{R}^{n}\right) \mid x^{\prime \prime} \in L^{2}\left([0,1], \mathbf{R}^{n}\right), x \text { satisfies }(3.16)-(3.17)\right\} .
$$

Define $A_{4}: D\left(A_{4}\right) \rightarrow X$ by $\left(A_{4} x\right)(t)=-x^{\prime \prime}(t)$. Using the results presented in [6, Sect. 7.1], it is possible to show that $A_{4}$ is self-adjoint in $X$ and $\sigma\left(A_{4}\right)=\sigma_{d}\left(A_{4}\right) \subset[0,+\infty)$. In particular, if $M=N=I_{n}$, we have $\sigma\left(A_{4}\right)=\sigma_{d}\left(A_{4}\right)=\left\{(2 k)^{2} \pi^{2} \mid k \in \mathbf{Z}\right\}$.

Set $\Phi(x)=\int_{0}^{1} V(t, x) d t$ for $x \in X$. Because of Proposition 3.2 and Proposition 3.13 the rest of the proof of Theorem 3.12 is similar to Theorem 3.1.

Proposition 3.13 For any $B \in L^{\infty}\left([0,1], \mathcal{L}_{s}\left(\mathbf{R}^{n}\right)\right)$, there exists $\epsilon_{0}>0$ such that for any $\epsilon \in$ $\left(0, \epsilon_{0}\right]$ we have

$$
\begin{aligned}
& v_{M}^{s}\left(B+\epsilon I_{n}\right)=0=v_{M}^{s}\left(B-\epsilon I_{n}\right), \\
& i_{M}^{s}\left(B-\epsilon I_{n}\right)=i_{M}^{s}(B), \\
& i_{M}^{s}\left(B+\epsilon I_{n}\right)=i_{M}^{s}(B)+v_{M}^{s}(B) .
\end{aligned}
$$

In particular, if $v_{M}^{s}(B)=0$, we have $i_{M}^{s}\left(B+\epsilon I_{n}\right)=i_{M}^{s}(B)$ for all $\epsilon \in\left(0, \epsilon_{0}\right]$.

Remark 4 Note that $L^{4}\left([0,1], \mathbf{R}^{n}\right) \subset L^{2}\left([0,1], \mathbf{R}^{n}\right), L^{2}\left([0,1], \mathbf{R}^{+}\right) \subset L^{1}\left([0,1], \mathbf{R}^{+}\right)$. If $M=N=$ $I_{n}, T=1, \bar{B}_{1}=0 I_{n}<\bar{B}_{2}=\alpha^{2} I_{n}<(2 \pi)^{2} I_{n}, l_{0} \in L^{4}\left([0,1], \mathbf{R}^{2 n}\right)$ and $\gamma \in L^{2}\left([0,1], \mathbf{R}^{+}\right)$, then Theorem 3.12 reduces to ([16], Theorem 3.6). Moreover, as $\bar{B}_{1}=(2 k \pi)^{2} I_{n}<\bar{B}_{2}=\alpha \pi^{2} I_{n}<$ $(2(k+1) \pi)^{2} I_{n}$ where $k \in \mathbf{N}$, then Theorem 3.12 reduces to ([16], Theorem 3.7). Clearly, our result generalizes Theorems 3.6-3.7 in [16].

Similarly to Theorem 3.4, we have the following.

Theorem 3.14 Under the hypotheses of Theorem 3.12, assume, in addition, that 
$\left(V_{5,1}\right) V^{\prime}(t, \theta)=\theta, V(t, \theta)=0$ and there exists $B_{01} \in L^{\infty}\left([0,1], \mathcal{L}_{s}\left(\mathbf{R}^{n}\right)\right)$ such that $B_{01} \geq \bar{B}_{1}$, $i_{M}^{s}\left(B_{01}\right)>i_{M}^{s}\left(\bar{B}_{1}\right)+v_{M}^{s}\left(\bar{B}_{1}\right)$, and (3.14) holds.

Then (3.15)-(3.17) has at least one nontrivial solution.

\section{Appendix}

In this section we recall some results from $[5,6]$ related to index theory for unbounded linear self-adjoint operator equations. Let $X$ be a real infinite-dimensional separable Hilbert space with norm $\|\cdot\|$ and inner product $(\cdot, \cdot)$. Let $A: D(A) \rightarrow X$ be unbounded self-adjoint and $\sigma(A)=\sigma_{d}(A)=\{\lambda \in \mathbf{R} \mid \lambda$ belongs to the point spectrum of $A\}$. For any $B_{1}, B_{2} \in \mathcal{L}_{s}(X)$, we write $B_{1}<B_{2}$ with respect to $X_{1}$ (a subspace of $X$ ) if and only if $\left(B_{1} x, x\right)<\left(B_{2} x, x\right)$ for all $x \in X_{1} \backslash\{\theta\}$; and write $B_{1} \leq B_{2}$ w.r.t. $X_{1}$ if and only if $\left(B_{1} x, x\right) \leq\left(B_{2} x, x\right)$ for all $x \in X_{1}$. If $X=X_{1}$ we just write $B_{1}<B_{2}$ or $B_{1} \leq B_{2}$.

Definition A.1 ([5], Definition 3.1.1) For any $B \in \mathcal{L}_{s}(X)$, define

$$
v_{A}(B)=\operatorname{dim} \operatorname{ker}(A-B) .
$$

$\nu_{A}(B)$ is called the nullity of $B$, where $\operatorname{dim} \operatorname{ker}(A-B)=\operatorname{dimension}$ of $\operatorname{ker}(A-B)$ and $\operatorname{ker}(A-$ $B)=\{x \in X \mid A x=B x\}$.

It was proved in [5] that the nullity $v_{A}(B)$ is finite.

Definition A.2 ([5], Definition 3.1.2) For any $B_{1}, B_{2} \in \mathcal{L}_{s}(X)$ with $B_{1}<B_{2}$, define

$$
I_{A}\left(B_{1}, B_{2}\right)=\sum_{\lambda \in[0,1)} v_{A}\left((1-\lambda) B_{1}+\lambda B_{2}\right)
$$

and, for any $B_{1}, B_{2} \in \mathcal{L}_{s}(X)$, define

$$
I_{A}\left(B_{1}, B_{2}\right)=I_{A}\left(B_{1}, k \mathrm{Id}\right)-I_{A}\left(B_{2}, k \mathrm{Id}\right),
$$

where Id : $X \rightarrow X$ is the identity map and $k \mathrm{Id}>B_{1}, k \mathrm{Id}>B_{2}$ for some real number $k>0$.

Let $B_{0} \in \mathcal{L}_{s}(X)$ be fixed and let $i_{A}\left(B_{0}\right)$ be a prescribed integer associated with $B_{0}$.

Definition A.3 ([5], Definition 3.1.3) For any $B \in \mathcal{L}_{s}(X)$, define

$$
i_{A}(B)=i_{A}\left(B_{0}\right)+I\left(B_{0}, B\right) .
$$

$i_{A}(B)$ is called the index of $\mathrm{B}$ and $i_{A}\left(B_{0}\right)$ is called initial index. Generally, the initial index can be any prescribed integer and the index $i_{A}(B)$ depends on $B_{0}$ and the initial index.

Proposition A.4 ([5], Proposition 3.1.5)

(1) For any $B, B_{1}, B_{2} \in \mathcal{L}_{s}(X), I_{A}\left(B_{1}, B_{2}\right)$ and $i_{A}(B)$ are well-defined and finite;

(2) for any $B_{1}, B_{2} \in \mathcal{L}_{s}(X), I_{A}\left(B_{1}, B_{2}\right)=i_{A}\left(B_{2}\right)-i_{A}\left(B_{1}\right)$;

(3) for any $B_{1}, B_{2}, B_{3} \in \mathcal{L}_{s}(X), I_{A}\left(B_{1}, B_{2}\right)+I_{A}\left(B_{2}, B_{3}\right)=I_{A}\left(B_{1}, B_{3}\right)$;

(4) for any $B_{1}, B_{2} \in \mathcal{L}_{s}(X)$, if $B_{1}<B_{2}$, then $v_{A}\left(B_{1}\right)+i_{A}\left(B_{1}\right) \leq i_{A}\left(B_{2}\right)$. 
Let $B_{0} \in \mathcal{L}_{s}(X)$ be given. From Lemma 3.2.1 in [5], we know that $A-B_{0}: Y \rightarrow X$ is continuous, $\operatorname{ker}\left(A-B_{0}\right)$ is finite-dimensional, $R\left(A-B_{0}\right)$ is closed and $X=\operatorname{ker}\left(A-B_{0}\right) \oplus$ $R\left(A-B_{0}\right)$, and the operator $\Lambda_{0}:=\left.\left(A-B_{0}\right)\right|_{R\left(A-B_{0}\right)}: R\left(A-B_{0}\right) \cap Y \rightarrow R\left(A-B_{0}\right)$ is invertible and the inverse $\Lambda_{0}^{-1}: R\left(A-B_{0}\right) \rightarrow R\left(A-B_{0}\right) \cap Y \hookrightarrow R\left(A-B_{0}\right)$ is compact and self-adjoint. For any $B \in \mathcal{L}_{s}(X)$ with $B-B_{0} \geq \epsilon$ for some constant $\epsilon>0$, we define a bilinear form:

$$
q_{A, B \mid B_{0}}(x, y)=-\left(\Lambda_{0}^{-1} x, y\right)+\left(\left(B-B_{0}\right)^{-1} x, y\right), \quad \forall x, y \in R\left(A-B_{0}\right) .
$$

By Proposition 3.2.2 in [5] we have

$$
R\left(A-B_{0}\right)=E_{A}^{+}\left(B \mid B_{0}\right) \oplus E_{A}^{0}\left(B \mid B_{0}\right) \oplus E_{A}^{-}\left(B \mid B_{0}\right),
$$

such that $q_{A, B \mid B_{0}}$ is positive definite, null and negative definite on $E_{A}^{+}\left(B \mid B_{0}\right), E_{A}^{0}\left(B \mid B_{0}\right)$ and $E_{A}^{-}\left(B \mid B_{0}\right)$, respectively. Moreover, $E_{A}^{0}\left(B \mid B_{0}\right)$ and $E_{A}^{-}\left(B \mid B_{0}\right)$ are finite-dimensional.

Definition A.5 ([5], Definition 3.2.3) For any $B \in \mathcal{L}_{s}(X)$ with $B-B_{0} \geq \epsilon$ for some constant $\epsilon>0$, we define

$$
i_{A}\left(B \mid B_{0}\right)=\operatorname{dim} E_{A}^{-}\left(B \mid B_{0}\right), v_{A}\left(B \mid B_{0}\right)=\operatorname{dim} E_{A}^{0}\left(B \mid B_{0}\right) .
$$

This relative index is a kind of Morse index. It plays an important role in the relationship between Morse index and the index $\left(i_{A}(B), v_{A}(B)\right)$.

Lemma A.6 ([5], Theorem 3.2.4) For any $B \in \mathcal{L}_{s}(X)$ with $B-B_{0} \geq \epsilon$ for some constant $\epsilon>0$, we have

(1) $v_{A}\left(B \mid B_{0}\right)=v_{A}(B)$

(2) $i_{A}\left(B \mid B_{0}\right)=I_{A}\left(B_{0}, B\right)-v_{A}\left(B_{0}\right)$.

As a first example, consider the following first order Hamiltonian system:

$$
\begin{aligned}
& -J \dot{x}=B(t) x, \\
& x_{1}(0) \cos \alpha+x_{2}(0) \sin \alpha=0, \\
& x_{1}(1) \cos \beta+x_{2}(1) \sin \beta=0,
\end{aligned}
$$

where $B \in L^{\infty}\left([0,1], \mathcal{L}_{s}\left(\mathbf{R}^{2 n}\right)\right), 0 \leq \alpha<\pi$ and $0<\beta \leq \pi, x=\left(x_{1}, x_{2}\right) \in \mathbf{R}^{n} \times \mathbf{R}^{n}, J=$ $\left(\begin{array}{cc}0 & -I_{n} \\ I_{n} & 0\end{array}\right)$. Let $X=L^{2}\left([0,1], \mathbf{R}^{2 n}\right), D(A)=\left\{x \in H^{1}\left([0,1], \mathbf{R}^{2 n}\right) \mid x\right.$ satisfies (A.4)-(A.5) $\}$. Define $A: D(A) \rightarrow X$ by $(A x)(t)=-J \dot{x}(t)$. It is easy to check that $A$ is self-adjoint and $\sigma(A)=$ $\sigma_{d}(A)=\{\beta-\alpha+k \pi \mid k \in \mathbf{Z}\}$. For any $B_{1}, B_{2} \in L^{\infty}\left([0,1], \mathcal{L}_{s}\left(\mathbf{R}^{2 n}\right)\right)$, we write $B_{1} \leq B_{2}$ if $B_{1}(t) \leq B_{2}(t)$, for a.e. $t \in[0,1]$; and we write $B_{1}<B_{2}$ if $B_{1}(t) \leq B_{2}(t)$ and $B_{1}(t)<B_{2}(t)$ on a subset of $[0,1]$ with positive measure.

Definition A.7 ([5], Definition 3.4.1) For any $B \in L^{\infty}\left([0,1], \mathcal{L}_{s}\left(\mathbf{R}^{2 n}\right)\right)$, we define

$$
\begin{aligned}
& v_{\alpha, \beta}^{f}(B)=\operatorname{dim} \operatorname{ker}(A-B), \\
& i_{\alpha, \beta}^{f}\left(\operatorname{diag}\left\{0, I_{n}\right\}\right)=i_{I_{n}, \alpha, \beta}^{s}(0),
\end{aligned}
$$




$$
i_{\alpha, \beta}^{f}(B)=i_{\alpha, \beta}^{f}\left(\operatorname{diag}\left\{0, I_{n}\right\}\right)+I_{\alpha, \beta}^{f}\left(\operatorname{diag}\left\{0, I_{n}\right\}, B\right),
$$

and

$$
\begin{aligned}
& I_{\alpha, \beta}^{f}\left(B_{1}, B_{2}\right)=\sum_{\lambda \in[0,1)} v_{\alpha, \beta}^{f}\left((1-\lambda) B_{1}+\lambda B_{2}\right) \quad \text { as } B_{1}<B_{2}, \\
& I_{\alpha, \beta}^{f}\left(B_{1}, B_{2}\right)=I_{\alpha, \beta}^{f}\left(B_{1}, k I d\right)-I_{\alpha, \beta}^{f}\left(B_{2}, k I d\right),
\end{aligned}
$$

for every $B_{1}, B_{2}$ with $k I d>B_{1}, k I d>B_{2}$.

Proposition A.8 ([5], Proposition 3.4.2; [6], Proposition 8.1.1)

(1) For any $B \in L^{\infty}\left([0,1], \mathcal{L}_{s}\left(\mathbf{R}^{2 n}\right)\right), v_{\alpha, \beta}^{f}$ is the dimension of the solution subspace of (A.3)-(A.5) and

$$
\left(i_{\alpha, \beta}^{f}(B), v_{\alpha, \beta}^{f}(B)\right) \in \mathbf{Z} \times\{0,1,2, \ldots, n\} .
$$

(2) For any $B_{1}, B_{2} \in L^{\infty}\left([0,1], \mathcal{L}_{s}\left(\mathbf{R}^{2 n}\right)\right)$, if $B_{1} \leq B_{2}$, then $i_{\alpha, \beta}^{f}\left(B_{1}\right) \leq i_{\alpha, \beta}^{f}\left(B_{2}\right)$ and $i_{\alpha, \beta}^{f}\left(B_{1}\right)+v_{\alpha, \beta}^{f}\left(B_{1}\right) \leq i_{\alpha, \beta}^{f}\left(B_{2}\right)+v_{\alpha, \beta}^{f}\left(B_{2}\right) ;$ if $B_{1}<B_{2}$, then $i_{\alpha, \beta}^{f}\left(B_{1}\right)+v_{\alpha, \beta}^{f}\left(B_{1}\right) \leq i_{\alpha, \beta}^{f}\left(B_{2}\right)$.

(3) For any $B \in L^{\infty}\left([0,1], \mathcal{L}_{s}\left(\mathbf{R}^{n}\right)\right)$,

$$
\left(v_{\alpha, \beta}^{s}(B), i_{\alpha, \beta}^{s}(B)\right)=\left(v_{\alpha, \beta}^{f}\left(\operatorname{diag}\left\{B, I_{n}\right\}\right), i_{\alpha, \beta}^{f}\left(\operatorname{diag}\left\{B, I_{n}\right\}\right)\right) .
$$

As a second example, consider the following first order Hamiltonian system:

$$
\begin{aligned}
& -J \dot{x}=B(t) x, \\
& x(1)=P x(0),
\end{aligned}
$$

where $P$ is symplectic, i.e., $P^{T} J P=J$. Let $X=L^{2}\left([0,1], \mathbf{R}^{2 n}\right), D(A)=\left\{x \in H^{1}\left([0,1], \mathbf{R}^{2 n}\right) \mid\right.$ $x$ satisfies (A.7)\}. Define $A: D(A) \rightarrow X$ by $(A x)(t)=-J \dot{x}(t)$. It is easy to check that $A$ is selfadjoint and $\sigma(A)=\sigma_{d}(A)=\left\{\mu_{i}+2 j \pi \mid i=1,2, \ldots, m ; j \in \mathbf{Z}\right\}$, where $\left\{\mu_{i}\right\}_{i=1}^{m} \subseteq(0,2 \pi]$ such that, for some non-zero $\xi_{i} \in \mathbf{R}^{2 n},\left(e^{-J \mu_{i}} P-I_{2 n}\right) \xi_{i}=0$ and $\left(\xi_{i}, \xi_{j}\right)_{\mathbf{R}^{2 n}}=0$ if $\mu_{i}=\mu_{j}$ for $i \neq j$.

Definition A.9 ([5], Definition 3.5.1) For any $B \in L^{\infty}\left([0,1], \mathcal{L}_{s}\left(\mathbf{R}^{2 n}\right)\right)$, we define

$$
\begin{aligned}
& v_{P}^{f}(B)=\operatorname{dim} \operatorname{ker}(A-B), \\
& i_{P}^{f}(B)=i_{P}^{f}(0)+I_{P}^{f}(0, B),
\end{aligned}
$$

and

$$
\begin{aligned}
& I_{P}^{f}\left(B_{1}, B_{2}\right)=\sum_{\lambda \in[0,1)} v_{P}^{f}\left((1-\lambda) B_{1}+\lambda B_{2}\right) \quad \text { as } B_{1}<B_{2}, \\
& I_{P}^{f}\left(B_{1}, B_{2}\right)=I_{P}^{f}\left(B_{1}, k I d\right)-I_{P}^{f}\left(B_{2}, k I d\right),
\end{aligned}
$$

for every $B_{1}, B_{2}$ with $k I d>B_{1}, k I d>B_{2}$.

Proposition A.10 ([5], Proposition 3.5.2)

(1) For any $B \in L^{\infty}\left([0,1], \mathcal{L}_{s}\left(\mathbf{R}^{2 n}\right)\right)$, we have $v_{P}^{f}(B) \in\{0,1,2, \ldots, 2 n\}$. 
(2) For any $B_{1}, B_{2} \in L^{\infty}\left([0,1], \mathcal{L}_{s}\left(\mathbf{R}^{2 n}\right)\right)$, if $B_{1} \leq B_{2}$, then $i_{P}^{f}\left(B_{1}\right) \leq i_{p}^{f}\left(B_{2}\right)$ and $i_{P}^{f}\left(B_{1}\right)+v_{P}^{f}\left(B_{1}\right) \leq i_{P}^{f}\left(B_{2}\right)+v_{P}^{f}\left(B_{2}\right) ;$ if $B_{1}<B_{2}$, then $i_{P}^{f}\left(B_{1}\right)+v_{P}^{f}\left(B_{1}\right) \leq i_{P}^{f}\left(B_{2}\right)$.

As a third example, consider the following second order Hamiltonian system:

$$
\begin{aligned}
& -\left(\Lambda(t) x^{\prime}\right)^{\prime}=B(t) x, \\
& x(0) \cos \alpha-\Lambda(0) x^{\prime}(0) \sin \alpha=0, \\
& x(1) \cos \beta-\Lambda(1) x^{\prime}(1) \sin \beta=0,
\end{aligned}
$$

where $\Lambda \in C\left([0,1], \mathcal{L}_{s}\left(\mathbf{R}^{n}\right)\right), \Lambda(t)$ is positive definite for $t \in[0,1], B \in L^{\infty}\left([0,1], \mathcal{L}_{s}\left(\mathbf{R}^{n}\right)\right)$, $0 \leq \alpha<\pi$ and $0<\beta \leq \pi$. Let $X=L^{2}\left([0,1], \mathbf{R}^{n}\right), D(A)=\left\{x \in C^{2}\left([0,1], \mathbf{R}^{n}\right) \mid\left(\Lambda(t) x^{\prime}\right)^{\prime} \in\right.$ $L^{2}\left([0,1], \mathbf{R}^{n}\right), x$ satisfies (A.9)-(A.10) $\}$. Define $A: D(A) \rightarrow X$ by $(A x)(t)=-\left(\Lambda(t) x^{\prime}(t)\right)^{\prime}$. From Sect. 3.3 in [5], we can check that $A$ is self-adjoint and $\sigma(A)=\sigma_{d}(A)$. In particular, if $\Lambda(t)=I_{n}, \alpha=0, \beta=\pi$, we have $\sigma(A)=\sigma_{d}(A)=\left\{k^{2} \pi^{2} \mid k \in \mathbf{Z}\right\}$.

Definition A.11 ([5], Sect. 3.3) For any $B \in L^{\infty}\left([0,1], \mathcal{L}_{s}\left(\mathbf{R}^{n}\right)\right)$, we define

$$
\begin{aligned}
& v_{\Lambda, \alpha, \beta}^{s}(B)=\operatorname{dim} \operatorname{ker}(A-B), \\
& i_{\Lambda, \alpha, \beta}^{s}(B)=\sum_{\lambda>0} v_{\Lambda, \alpha, \beta}^{s}\left(B+\lambda I_{n}\right) .
\end{aligned}
$$

Proposition A.12 ([5], Propositions 2.3.3 and 3.1.5)

(1) For any $B \in L^{\infty}\left([0,1], \mathcal{L}_{s}\left(\mathbf{R}^{n}\right)\right)$, we have $v_{\Lambda, \alpha, \beta}^{s} \in\{0,1,2, \ldots, n\}$.

(2) For any $B_{1}, B_{2} \in L^{\infty}\left([0,1], \mathcal{L}_{s}\left(\mathbf{R}^{n}\right)\right)$, if $B_{1} \leq B_{2}$, then $i_{\Lambda, \alpha, \beta}^{s}\left(B_{1}\right) \leq i_{\Lambda, \alpha, \beta}^{s}\left(B_{2}\right)$ and $i_{\Lambda, \alpha, \beta}^{s}\left(B_{1}\right)+v_{\Lambda, \alpha, \beta}^{s}\left(B_{1}\right) \leq i_{\Lambda, \alpha, \beta}^{s}\left(B_{2}\right)+v_{\Lambda, \alpha, \beta}^{s}\left(B_{2}\right) ;$ if $B_{1}<B_{2}$, then $i_{\Lambda, \alpha, \beta}^{s}\left(B_{1}\right)+v_{\Lambda, \alpha, \beta}^{s}\left(B_{1}\right) \leq i_{\Lambda, \alpha, \beta}^{s}\left(B_{2}\right)$.

\section{Acknowledgements}

The authors would like to thanks the anonymous reviewers very much for their positive comments, careful reading and useful suggestions on improving this article.

\section{Funding}

The authors are partially supported by the National Natural Science Foundation of China (Grant No.11601193; No.11901248) and the Natural Science Foundation of the Jiangsu Higher Education Institutions of China (No.18KJB1 10006). Meanwhile, the second author is partially supported by the QingLan Project of Jiangsu Province of China.

Availability of data and materials

Data sharing is not applicable to this article as no datasets were generated or analysed during the current study.

\section{Competing interests}

The authors declare that they have no competing interests.

Authors' contributions

All authors contributed equally and significantly in writing this paper and typed, read, and approved the final manuscript.

\section{Publisher's Note}

Springer Nature remains neutral with regard to jurisdictional claims in published maps and institutional affiliations. 


\section{References}

1. Chang, K.C., Guo, M.: Lecture Notes on Functional Analysis (II). Peking University Press, Beijing (1990) (in Chinese)

2. Clarke, F.H., Ekeland, I.: Nonlinear oscillations and boundary value problems for Hamiltonian systems. Arch. Ration. Mech. Anal. 78(4), 315-333 (1982)

3. Dong, Y.J: Maslov-type index theory for linear Hamiltonian systems with Bolza boundary value conditions and multiple solutions for nonlinear Hamiltonian systems. Pac. J. Math. 221, 253-280 (2005)

4. Dong, Y.J.: P-index theory for linear Hamiltonian systems and multiple solutions for nonlinear Hamiltonian systems. Nonlinearity 19, 1275-1294 (2006)

5. Dong, Y.J.: Index theory for linear self-adjoint operator equations and nontrivial solutions for asymptotically linear operator equations. Calc. Var. 38, 75-109 (2010)

6. Dong, Y.J.: Index Theory for Hamiltonian Systems and Multiple Solution Problems. Science Press, Beijing (2014)

7. Ekeland, I:: Convexity Methods in Hamiltonian Mechanics. Springer, Berlin (1990)

8. Ekeland, I., Temam, R.: Convex Analysis and Variational Problems. North-Holland, Amsterdam (1976)

9. Fonda, A., Ramos, M., Willem, M.: Subharmonic solutions for second-order differential equations. Topol. Methods Nonlinear Anal. 1(1), 49-66 (1993)

10. Hirano, N.: Subharmonic solutions for second-order differential systems. J. Math. Anal. Appl. 196(1), 266-281 (1995)

11. Hirano, N., Wang, Z.Q.: Subharmonic solutions for second-order Hamiltonian systems. Discrete Contin. Dyn. Syst. 4(3), 467-474 (1998)

12. Jiang, Q., Tang, C.L.: Periodic and subharmonic solutions of a class of subquadratic second-order Hamiltonian systems. J. Math. Anal. Appl. 328(1), 380-389 (2007)

13. Long, Y.M.: Nonlinear oscillations for classical Hamiltonian systems with bi-even subquadratic potentials. Nonlinear Anal. 24, 1665-1671 (1995)

14. Long, Y.M.: Index Theory for Symplectic Paths with Applications. Birkhäuser, Boston (2002)

15. Mawhin, J., Willem, M.: Critical points of convex perturbations of some indefinite quadratic forms and semi-linear boundary value problems at resonance. Ann. Inst. Henri Poincaré, Anal. Non Linéaire 6, 431-453 (1986)

16. Mawhin, J., Willem, M.: Critical Point Theory and Hamiltonian Systems. Springer, Berlin (1989)

17. Rabinowitz, P.H.: On subharmonic solutions of Hamiltonian systems. Commun. Pure Appl. Math. 33(5), 609-633 (1980)

18. Tang, C.L., Wu, X.P.: Notes on periodic solutions of subquadratic second order systems. J. Math. Anal. Appl. 285, 8-16 (2003)

19. Tang, C.L., Wu, X.P.: Subharmonic solutions for nonautonomous sublinear second-order Hamiltonian systems. J. Math Anal. Appl. 304, 383-393 (2005)

20. Tang, C.L., Wu, X.P.: Some critical point theorems and their applications to periodic solution for second order Hamiltonian systems. J. Differ. Equ. 248, 660-692 (2010)

\section{Submit your manuscript to a SpringerOpen ${ }^{\circ}$ journal and benefit from:}

- Convenient online submission

- Rigorous peer review

- Open access: articles freely available online

- High visibility within the field

- Retaining the copyright to your article

Submit your next manuscript at $\gg$ springeropen.com 